\title{
Simulation of the Developed Electro-Thermal Aging Model based on Trapping and Detrapping Process
}

\author{
Hisham A. Alghamdi ${ }^{1,2}$, George Chen ${ }^{1}$, Alun S. Vaughan ${ }^{1}$ \\ 1)School of Electronics and Computer Science \\ University of Southampton, Southampton \\ SO17 1BJ, United Kingdom \\ 2) Engineering Department, Najran University \\ Najran, Saudi Arabia \\ Email: haalghamdi@nu.edu.sa
}

\begin{abstract}
The key aspect of this work is to simulate the evolution of structure damages in polyethylene under the effect of electro-thermal stresses based on the trapping and detrapping aging model. In addition, the susceptibility of the model parameters to the isolated sites during the aging period have also been studied. The simulation work is performed on a 2D square grid that is assumed to represent a part of the insulation. The mesh structure is divided using the finite element method. Based on the nature of polyethylene, its structure is semi-crystalline with a spatially varying morphology. Consequently, each bond in the grid is assigned a set of parameter values. One of these parameters is the critical fraction of trapped charges $C^{*}$, which needs to be reached in order to fail a bond. It is chosen at random values from a range centered on the characteristic value obtained from the experimental results. This indicates that the insulation life at varying parameter $C^{*}$ is lower than its characteristic value.
\end{abstract}

Keywords-Ageing, Lifetime, Electro-thermal Breakdown, Space Charge, Decay, Conductivity, Schottky, Trap, Detrap, LDPE

\section{INTRODUCTION}

The work presented in this paper investigates the aging process by describing the growth of breakdown path. Generally, it was assumed during the aging process that molecule dissociation contributes to change in the structure of polymer such as changes in morphology leading to the final breakdown [1]-[3]. In a homogeneous material, molecule dissociation seems to randomly take place in the insulating material, and the final breakdown could occur due to the accumulation of the deteriorated regions with molecule dissociations. Some of the models used the percolation process to describe the accumulation of deteriorated regions as shown in [4]. Other simulation models of mechanical failure assumed that the crack development during aging was not only due to the growth itself, but also due to the accumulation of damage regions [5], [6]. However, because the failure was assumed to be only due to the formation of percolation path through the insulation, these models are not suitable for use in electrical aging processes where the breakdown strength has to be gradually decreased over the time of the aging process.

It is important to mention that the insulation below a critical value of stress should infinitely survive. This is the definition of deterministic breakdown theories [7]. In the developed aging model adopted in this paper, we assume that the insulation systems below such critical values are well. Thus, the risk to its integrity is reduced as the local fields that lead to rapid premature failures are eliminated.

A great deal of effects have been developed to study the aging of polymeric insulations without explicit agreement among who as to which physical mechanism should be involved. One of the main concerns is the basis on which the model's parameters were chosen. Most of the aging models have a range of values for each parameter, but only the characteristic or average value is used in the model to extrapolate the life of a specific material. Therefore, it is convenient to study the effects of the model parameters by representing the insulator as a grid whose bonds are identical capacitors. Each individual capacitor can have a different random number within the range of the upper and lower values of the model's parameter. For example, electric fields at some regions are higher, based on the concentration of charges at these regions, also spatial location of chemical structures within the polymer is different, which causes variation in the depth of traps. These assumptions will not only affect the rate at which a single defect grows up, but also to defect amalgamation during cluster generation and the formation of breakdown path.

\section{Aging Model Theory}

\section{A. Trapping Rate}

The trapping rate depends on the concentration of electronic charges which is proportional to the injected current density. The dynamic of trapping rate can be written as:

$$
\frac{d n_{T \rightarrow}}{d t}=K_{T}\left(N_{T}-n_{T}\right)
$$

where $N_{T}$ and $n_{T}$ are the total traps and the trapped charges, respectively. The trapping coefficient $K_{T}$ is proportional with the injected current density $(J)$ as shown in [8], [9] as

$$
K_{T}=\frac{J \sigma_{T}}{q}
$$

where $q$ is the charge amount and $\sigma_{T}$ is the capture crosssection area.

For simplicity purposes, charge carriers in insulation materials are assumed to be generated via charge injection from the 
electrodes. Some of the injection mechanisms are reported in the literature such as the Schottky effect and tunneling [10]. To identify the appropriate mechanism in our system, the values of measured current densities versus the applied electric fields have been traced. The Schottky injection mechanism fits well with the experimental data for low-density polyethylene LDPE as shown in Figure 1. It is given as:

$$
J=A^{*} T^{2} \exp \left(-\frac{\Phi-\beta_{s} E^{1 / 2}}{k T}\right)
$$

where $\Phi$ is the barrier height of the electrode-insulation barrier, $\beta_{s}=\left(q / 4 \pi \varepsilon_{0} \varepsilon_{r}\right)^{1 / 2}$ and $A^{*}$ is called the effective Richardson-Dushman constant, which is a function of the effective mass of electron [11] as:

$$
A^{*}=\frac{4 \pi q m^{*} k^{2}}{h^{3}}
$$

where $h$ is the Planck constant and $m^{*}$ is the effective mass.

\section{B. Detrapping Rate}

Once the electron has been captured, different mechanisms can cause its detrapping [12]. These mechanisms are (1) photon assisted depopulation, which is negligible in the present study as the sample was not exposed to any light, (2) impact ionization and (3) tunneling. The latter two take place at very high electric fields and are not considered in this work, and (4) thermal detrapping. Only the latter is considered as a detrapping mechanism in this paper, and this occurs when the trapped charge carrier gains its energy for detrapping from the thermal lattice vibrations. The mathematical expression for the detrapping process can be described as:

$$
\frac{d n_{T \leftarrow}}{d t}=-K_{D} n_{T}
$$

where $K_{D}$ is the detrapping coefficient. This coefficient was used for detrapping in the literature, with good fitting results [13]-[15]. It is expressed as:

$$
K_{D}=N_{c} v_{t h} \sigma_{T} \exp \left(-\frac{E_{T}-W_{e m}}{k T}\right)
$$

where $N_{c}$ is the effective density of energy states in the conduction band, $v_{t h}$ is the thermal velocity of the charge, $E_{T}$ is the trap depth, $k$ the Boltzmann constant and $T$ is the absolute temperature. The stored electromechanical energy, $W_{e m}$, in the spherical shell can be estimated as:

$$
W_{e m}=\frac{\sigma_{e m}\left(r_{0}\right)^{2}}{2 Y} V
$$

where $V$ is the spherical volume, $Y$ is Young's modulus which is roughly equal to $1.5 \times 10^{8} \mathrm{~N} / \mathrm{m}^{2}$ for polyethylene. The electromechanical stress, $\sigma_{e m}$, is given as:

$$
\sigma_{e m}\left(r_{0}\right)=\frac{\varepsilon_{0} \varepsilon_{r} E\left(r_{0}\right)^{2}}{2}
$$

where $\varepsilon_{0}$ and $\varepsilon_{r}$ are the permittivity of free space and the relative permittivity of polyethylene, respectively.

\section{Kinetics of the Trapping and Detrapping Process}

Based on the above explanation, the kinetics of the trapping and detrapping process can be expressed as the sum of $\left(d n_{T \rightarrow} / d t\right)$ and $\left(d n_{T \leftarrow} / d t\right)$, it yields:

$$
\frac{d n_{T}}{d t}=K_{T}\left(N_{T}-n_{T}\right)-K_{D} n_{T}
$$

We can see that Eq.9 is a standard first order deferential equation. It can be solved if the initial condition is known. Before the injection takes place, the total quantity of trapped charges is zero $\left(n_{T}(x, 0)=0\right)$. Therefore, the solution is:

$$
n_{T}(t)=\frac{N_{T} K_{T}}{K_{T}+K_{D}}\left\{1-\exp \left[-\left(K_{T}+K_{D}\right) t\right]\right\}
$$

If we assume that the ratio of the trapped charges to the total traps can be described through a normalized variable $\mathrm{X}$ as:

$$
X=\frac{n_{t}}{N_{T}}
$$

then Eq.10 becomes:

$$
\begin{aligned}
X(t) & =C\left\{1-\exp \left[-\left(K_{T}+K_{D}\right) t\right]\right\} \\
& +C_{0} \exp \left[-\left(K_{T}+K_{D}\right) t\right]
\end{aligned}
$$

where $C_{0}$ is the initial value of $C$. This is the general form of the solution where $C_{0}$ was chosen to be zero in Eq.10 at specific conditions, and

$$
\begin{aligned}
C & =\frac{K_{T}}{K_{T}+K_{D}} \\
& =\left\{1+\frac{q N_{c} v_{t h}}{A^{*} T^{2}} \exp \left[-\frac{\left(E_{T}-W_{e m}\right)-\left(\Phi-\beta_{S} E^{1 / 2}\right)}{k T}\right]\right\}^{-1}
\end{aligned}
$$

is the equilibrium value of $X$.

From the definition of the variable $X$, all the traps are at the initial state (un-trapped) at $X=0$ and state of fully trapped charges at $X=1$. At a fraction of trapped charges, the released electromechanical energy from the detrapping process is able to break the insulation bonds and terminate the lifetime ( $\mathrm{t}$ in Eq.12). This fraction is symbolized here as $C^{*}$, which intails that the lifetime should never been terminated before the state of trapped charges reaches $C^{*}$.

The lifetime equation can be expressed by setting $X(T)$ equals to $C^{*}$ in the integrated equation, which gives:

$$
\begin{aligned}
t(E, T)= & {\left[\frac{\sigma_{T}}{q} A^{*} T^{2} \exp \left(-\frac{\Phi-\beta_{s} E^{1 / 2}}{k T}\right)\right.} \\
& \left.+N_{c} v_{t h} \sigma_{T} \exp \left(-\frac{E_{T}-W_{e m}}{k T}\right)\right]^{-1} \\
& \ln \left(\frac{\left.C(E)-C_{0}\right)}{C(E)-C^{*}}\right)
\end{aligned}
$$




\section{RESULTS AND DISCUSSION}

The results reported from simulations are performed on the applied fields ranging from $60 \mathrm{MV} / \mathrm{m}$ to $140 \mathrm{MV} / \mathrm{m}$ on a mesh grid of a bond that its length is $3.75 \mu \mathrm{m}$ at temperature of $80 C^{\circ}$. A set of the model parameter values is assigned for each bond. An investigation of the spatial variation in $C^{*}$ was carried out gives each bond a different value that was selected at random from a given range of values centered on the parameter value found to reproduce the characteristic value of the life distribution. The chosen range was from 0.48 to 0.20 , with the characteristic 0.34 as an average value. The trapping and detrapping was considered at the bond scale. A number of realizations have been considered for this variation range, and the evolution time that corresponds to broken bonds is plotted when $C^{*}$ is spatially distributed.

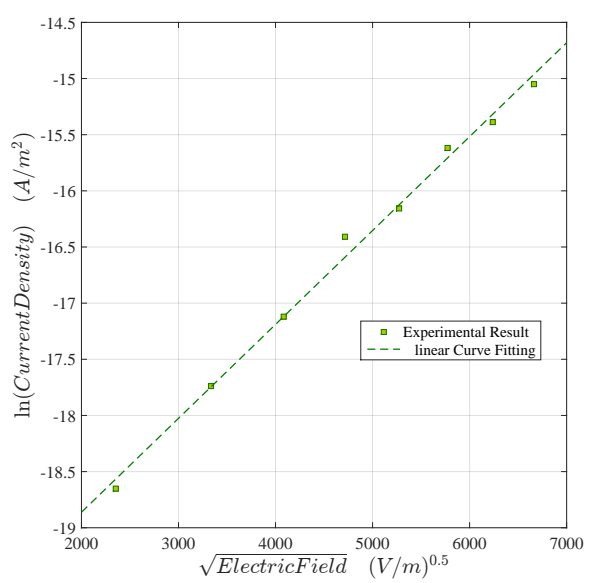

Fig. 1. Plot of square root of electric field versus $\ln$ of the current density to calculate the material permittivity of LDPE samples.

Initially, It is significant to explain how the parameter values have been chosen. From the literature, the effective density of states in the conduction band can be estimated as $N_{c}=2\left(2 \pi m_{e}{ }^{*} k T / h^{2}\right)^{3 / 2}$, where $m_{e}{ }^{*}$ is the electron effective mass which is roughly equal to $m_{e}$. For polyethylene, it is around the order of $10^{23}$ to $10^{25} \mathrm{~m}^{-3}$ [16]. The thermal velocity of the charge, $v_{t h}=\left(3 \mathrm{kT} / \mathrm{m}_{e}{ }^{*}\right)^{0.5}$, is in the order of $10^{5}$ $\mathrm{m} / \mathrm{s}$ [17]. The parameters of the Schottky effects (i.e., $\Phi$ and $A^{*}$ ) are extracted from the conductivity measurement of LDPE samples as shown in Figure 1. The relative permittivity $\varepsilon_{r}$ can be estimated from the slope of the curve as Slope $=\beta_{S} / k T$, while the $\Phi$ value can be estimated from the intercept of y-axis of the same curve as the rest of the parameters of the Schottky equation are known. On the other hand, the detrapping process is much simpler when the applied field is removed. Thus, the solution of Eq.9 becomes:

$$
n_{T}(t)=n_{0} \exp \left[-\left(K_{D}\right) t\right]
$$

where $n_{0}$ is the initial condition which can be obtained from Eq.10 at the moment where the applied field is removed. This can assist to extract the detrapping rate constant $K_{D}$ from the decay measurement of space charge in Figure 2. Substituting the detrapping rate constant in Eq.10, makes the trapping rate easy to be calculated. At this stage, the trap cross-section and the the trap depth can be calculated based on the trapping and detrapping rate constants. With regards to $C^{*}$, the value was extracted from Figure 3 using the curve fitting of Eq.14.

The way in which the simulation worked essentially began by distributing the electric field using the finite difference method (FDM). At the beginning of the simulation, we assumed that each bond is unfailed (similar to a non-conducting capacitor). The bond with the shortest time to failure by using Eq.14 was converted to conductor. Thereby, the local fields are changed at that time in the whole $2 \mathrm{D}$ grid. The amount of life lost in all of the other bonds is converted to an equivalent bonddependent value of $C_{0}$ appropriate to that time. Thereafter, repeating the same procedure to find the shortest lifetime bonds. On this simulation, there is no restriction on the number of broken bonds that should be reached to end the insulation life.

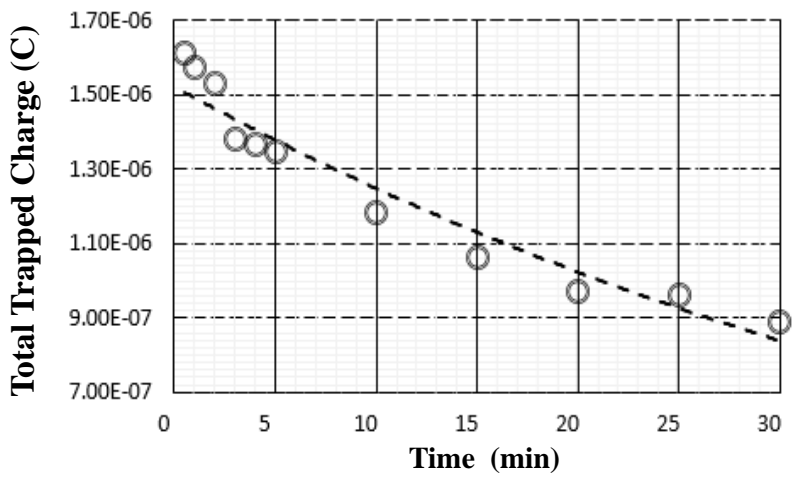

- Experimental result - - - - Expon. (Experimental result)

(a)

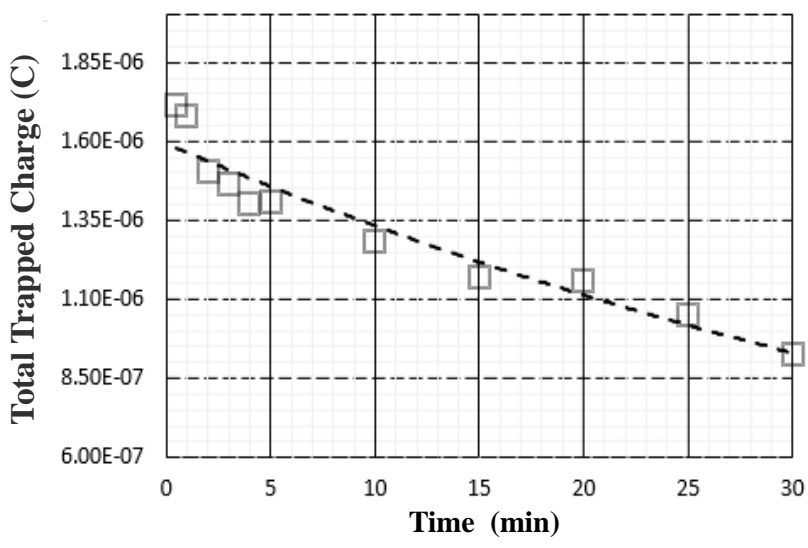

Experimental Result - - - - Expon. (Experimental Result)

Fig. 2. Decay of the total trapped charge after being stressed at $10 \mathrm{kV}$ for [a] 5 mins and [b] 3 mins.

During the aging process, an inspection of the damage affecting the sample shows that only isolated bonds are damaged. The unconnected bonds to the breakdown path can be obviously seen in Figure 4. Even though the values of $C^{*}$ for these bonds are small, which mean that they are most susceptible to damage, their capability for extension is limited. A sample failure is initiated by the irreversible damage to a bond where its local electric field is increased, and therefore 
increasing the mechanical energy that is produced in a trap. This mechanical energy is able to cause damage to the bond after being released by detraping an electron. The initiation of breaking bonds takes a considerable time, andthe damage extension is accelerated rapidly once one bond causes the neighbors to fail and connects the two electrodes via the conducting path, causing sample failure. Isolated damaged bonds lying next to or on the failure path are absorbed into the path. Some of these failed bonds cause minor deviation from a straight line. This pattern of behaviour is shown in all of the repeated simulations.

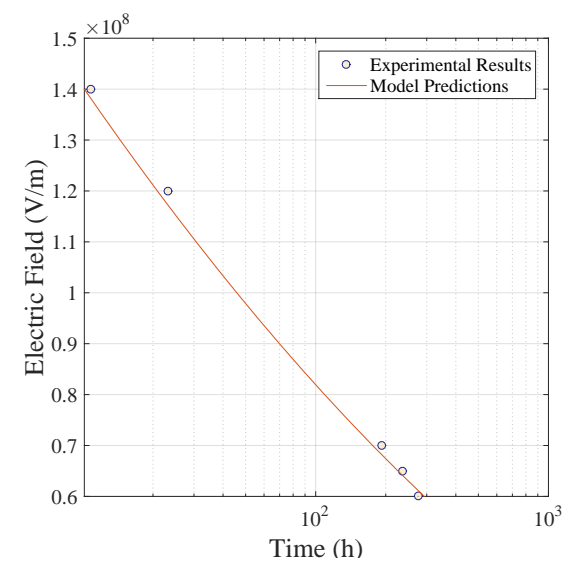

Fig. 3. The emperical lifetime for LDPE samples at different electric fields and fixed temperature of $80 C^{\circ}$.

Through the simulation results, the structure of isolated failures is similar to the expected form of a deterministic runaway process, where a filamentary conducting path is produced by a local reinforcement of damage generation [7]. This is roughly close to the evolution of the isolated failures in the simulation as shown in Figure 5. The slight difference in the simulation is that the beginning of runaway is considerably delayed rather than being initiated at the most susceptible site. The key factor in determining insulation life is not the susceptibility of individual local sites to damage, but the susceptibility of cluster sites together that leads to a selfreinforcing of extending damage. This conclusion is illustrated by the results from simulations when $C^{*}$ is given a spatial variation. A study of stochastic extension of the percolation model for breakdown showed similar results [18].

The simulation shows, in addition, that a reasonable number of isolated failed regions occur during the aging period as can be seen in Figure 4 before the breakdown process accelerates rapidly. However, this conclusion is in contrast to the study with [19] which states that a few of the bonds which failed during the main part of the aging period would be detectable as nano voids, cracks or local conducting regions [20]. The rest of the regions have not reached the critical situation for irreversibility as it is assumed that detecting the changes during the main part of aging period would be difficult.

The chosen lifetime at a specific field from repeated simulation results was deducted using the Weibull failure probability $\left(P_{F}\right)$, which is given as: [21]:

$$
P_{F}=1-\exp \left[-\left(\frac{t}{\alpha}\right)^{\beta}\right]
$$

where $\alpha$ is the characteristic life, and $\beta$ is the shape parameter of the distribution. Due to the limited number of sample tested for long aging, the experimental evaluation of the parameters $\alpha$ and $\beta$ still cannot be made precisely.

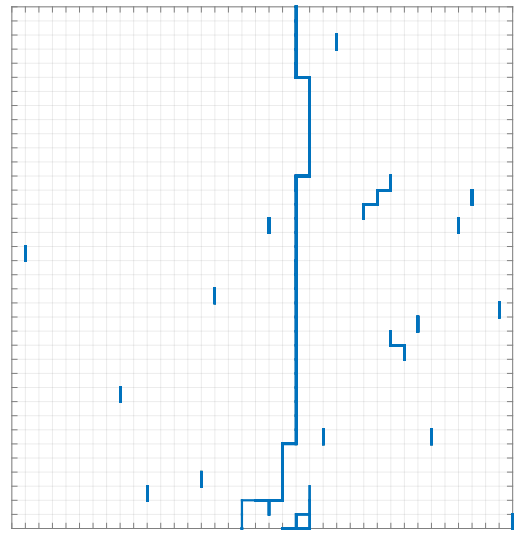

Fig. 4. Damage produced by failure corresponding to the damage evolution shown in Figure5.

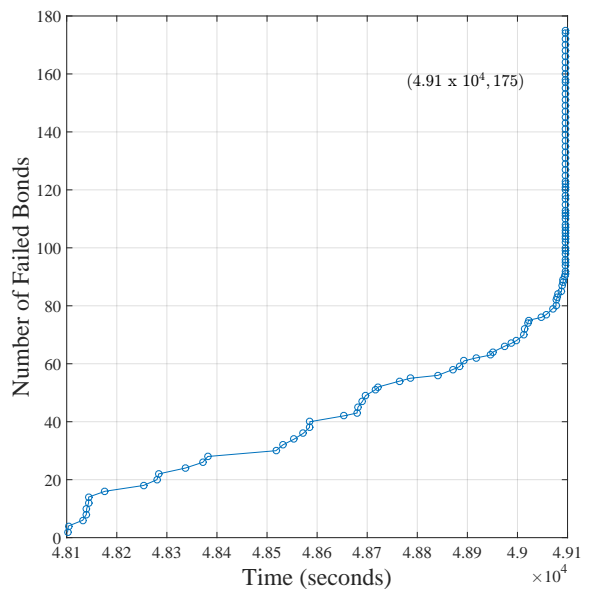

Fig. 5. The damage evolution during the aging process when electric field is $140 \mathrm{MV} / \mathrm{m}$, temperature is $80 C^{o}$ and spatial variation of $C^{*}$.

According to the results of lifetime from repeating simulations for a specific field, such as Figure 6, the variations among these results seems to be very small. This may be related to the fixed characteristic value for some parameters of the aging model which are not influenced during the simulation period, such as variation of trap depth. Here, we have assumed that five samples are sufficient to characterize the simulation lifetime for a chosen field since our aim is to investigate the effect of $C^{*}$ variations on the aging model. Moreover, the shape parameter of the distributed results, $\beta$, has a large value as the results are close to each other. This result, however, would be expected to be in contrast with what we can see from the empirical breakdown results. Figure 6 illustrates the Weibull distribution of several applied field with the variation of $C^{*}$. 


\begin{tabular}{ccc}
\hline Parameter & Unit & Value \\
\hline$\sigma_{T}$ & $m^{2}$ & $6.55 \times 10^{-16}$ \\
$N_{c}$ & $\mathrm{~cm}^{-3}$ & $3.21 \times 10^{+25}$ \\
$v_{t h}$ & $\mathrm{~ms}^{-1}$ & $1.27 \times 10^{+5}$ \\
$E_{T}$ & $\mathrm{~J}$ & $3.94 \times 10^{-19}$ \\
$\Phi$ & $\mathrm{J}$ & $2.70 \times 10^{-19}$ \\
$T$ & $\mathrm{~K}$ & 353 \\
$C^{*}$ & - & 0.34
\end{tabular}

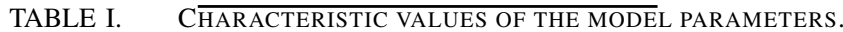

A suitable modification on the value of the fraction $C^{*}$ has been made to study its effect on the aging of polymer while the other parameters are fixed as shown in Table I. As an example, the characteristic lifetime of a LDPE sample stressed under $140 \mathrm{MV} / \mathrm{m}$ at a fixed value of $C^{*}$ is around 11 hours, while under the same conditions with $C^{*}$ ranging from 0.44 to 0.23 reducing to 7 hour, similarly the time at $60 \mathrm{MV} / \mathrm{m}$ is around 300 hours for the experimental work, and 200 hours with the variation of $C^{*}$. The reason could be that the most susceptible sites to aging with smallest values of $C^{*}$ can fail earlier than the expected time.

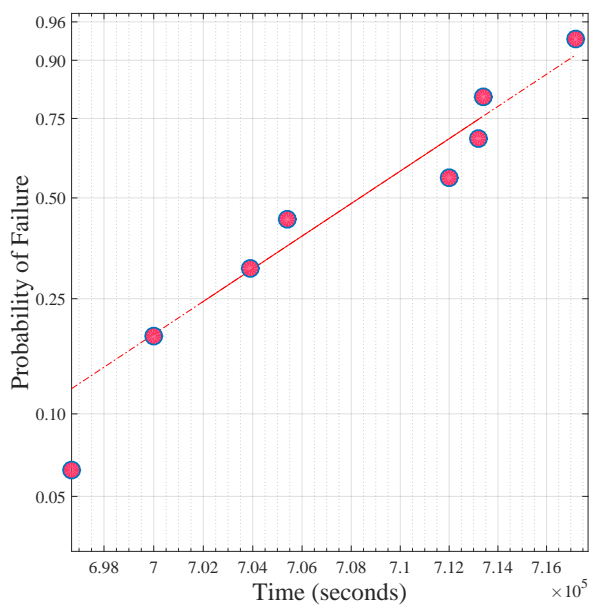

Fig. 6. Weibull plot of simulating lifetimes at a constant stress of $60 \mathrm{MV} / \mathrm{m}$ and $80 C^{\circ}$.

\section{CONCLUSION}

Simulations performed using the developed lifetime model with the spatial variation in the fraction of trapped charges $C^{*}$ yields a form of a single filamentary path as showing in the failure structure. A similarity to a deterministic runaway process has been found for the time evolution of the damage during the aging process, but the initiation of failing bonds is much more delayed. Damage occurs when sufficient morphological changes exist in a region that is able to continually increase the local energy concentration and therefore increasing the released energy from the detrapping process. Simulations performed at different applied fields cannot show a significant difference in the number of the isolated failures.

The lifetime distribution that results from the simulations can be fitted to the Weibull distribution. The shape parameter reflects the variation between the simulation results at the same field. It has been found to be a large value, which means repeated simulation results are close together.

\section{ACKNOWLEDGMENT}

The authors would like to thank the Ministry of Education in Saudi Arabia for the financial sponsorship .

\section{REFERENCES}

[1] T. Tanaka and A. Greenwood, "Effects of charge injection and extraction on tree initiation in polyethylene," Power Apparatus and Systems, IEEE Transactions on, no. 5, pp. 1749-1759, 1978.

[2] L. Dissado, G. Mazzanti, and G. Montanari, "The incorporation of space charge degradation in the life model for electrical insulating materials," Dielectrics and Electrical Insulation, IEEE Transactions on, vol. 2, no. 6, pp. 1147-1158, 1995.

[3] J.-L. Parpal, J.-P. Crine, and C. Dang, "Electrical aging of extruded dielectric cables. a physical model," Dielectrics and Electrical Insulation, IEEE Transactions on, vol. 4, no. 2, pp. 197-209, 1997.

[4] B. Halperin, S. Feng, and P. Sen, "Differences between lattice and continuum percolation transport exponents," Physical review letters, vol. 54, no. 22, p. 2391, 1985.

[5] W. Curtin and H. Scher, "Time-dependent damage evolution and failure in materials. mi. mtheory," Physical Review B, vol. 55, no. 18, p. 12038, 1997.

[6] M. I. Zeifman and D. Ingman, "A percolation model for lifetime variability in polymeric materials under creep conditions," Journal of Applied Physics, vol. 88, no. 1, pp. 76-87, 2000.

[7] L. A. Dissado and J. C. Fothergill, "Electrical degradation and breakdown in polymers," vol. 9, 1992.

[8] Y. Nissan-Cohen, J. Shappir, and D. Frohman-Bentchkowsky, "Dynamic model of trapping-detrapping in sio2," Journal of applied physics, vol. 58, no. 6, pp. 2252-2261, 1985.

[9] E. Avni and J. Shappir, "Modeling of charge-injection effects in metaloxide-semiconductor structures," Journal of applied physics, vol. 64, no. 2, pp. 734-742, 1988 .

[10] J. Simmons, "Conduction in thin dielectric films," Journal of Physics D: Applied Physics, vol. 4, no. 5, p. 613, 1971.

[11] M. Kiziroglou, X. Li, A. Zhukov, P. De Groot, and C. De Groot, "Thermionic field emission at electrodeposited ni-si schottky barriers," Solid-State Electronics, vol. 52, no. 7, pp. 1032-1038, 2008.

[12] C. Sah, "Models and experiments on degradation of oxidized silicon," Solid-state electronics, vol. 33, no. 2, pp. 147-167, 1990.

[13] J. Albohn, W. Füssel, N. Sinh, K. Kliefoth, and W. Fuhs, "Capture cross sections of defect states at the si/sio2 interface," Journal of Applied Physics, vol. 88, no. 2, pp. 842-849, 2000.

[14] Y. Wang and K. Cheung, "Carrier capture at the sio 2-si interface: A physical model," Applied Physics Letters, vol. 91, no. 11, pp. 113 509113509,2007

[15] D. Goguenheim, D. Vuillaume, G. Vincent, and N. M. Johnson, "Accurate measurements of capture cross sections of semiconductor insulator interface states by a trap-filling experiment: The chargepotential feedback effect," Journal of Applied Physics, vol. 68, no. 3, pp. 1104-1113, 1990.

[16] D. D. Gupta and M. Barbarez, "On electronic conduction in polyethylene films," Journal of Physics D: Applied Physics, vol. 6, no. 7, p. 867, 1973.

[17] K. Kao and W. Hwang, "Electronic transport in solids, international series in the science of the solid state, vol. 14. general editor br pamplin," 1981.

[18] K. Wu and Y. Cheng, "Simulation on the time dependence of breakdown strength in insulating polymers," Journal of applied physics, vol. 101, no. 6, p. 064113, 2007.

[19] L. A. Dissado and A. Thabet, "Simulation of electrical ageing in insulating polymers using a quantitative physical model," Journal of Physics D: Applied Physics, vol. 41, no. 8, p. 085412, 2008.

[20] L. Dissado, A. Thabet, and S. Dodd, "Simulation of dc electrical ageing in insulating polymer films," Dielectrics and Electrical Insulation, IEEE Transactions on, vol. 17, no. 3, pp. 890-897, 2010.

[21] "Ieee guide for the statistical analysis of electrical insulation breakdown data," IEEE Std 930-2004 (Revision of IEEE Std 930-1987), pp. 1-41, 2005. 